\title{
Predicting Falls in Healthy Elderly: A Comparison between Unified Balance Scale versus Four-Square Step Test
}

\author{
Arwa Sinnarwala ${ }^{1}$, Snehal Joshi ${ }^{2}$ \\ ${ }^{1}$ PG Student, Department of Neuro Physiotherapy, D.E.Society's Brijlal Jindal College of Physiotherapy, Pune. \\ ${ }^{2}$ Professor, Department of Neuro Physiotherapy, D.E.Society's Brijlal Jindal College of Physiotherapy, Pune.
}

Corresponding Author: Arwa Sinnarwala

\begin{abstract}
Background: Falls is a major incidence in elderly life and its sequence may be a major cause of mortality. Falls can be predicted and thus prevented. Screening scales are either a brief one component balance or more composite multifactorial assessment. Four Square Step test is an easy, less time consuming, versus Unified Balance scale which is multi domain. The present study was intended to see which of the two scales could accurately predict adults who were at risk of falls. Thus, helping therapist perform a single test to screen them and give them targeted treatment.

Method: 80 Healthy elderly above the age of 60 years were included in the study. Fall history was noted post which Four Square Step test and Unified Balance Scale was administered in sequence. Data Analysis was done, and sensitivities and specificities were calculated.

Result: Statistical Analysis was done by plotting the contingency table and plotting the Receiver Operating Curve. The Unified Balance Scale had a better combination of sensitivity and specificity than Four Square step test.

Conclusion: Unified Balance scale is better than Four-Square step test in predicting falls in healthy elderly.
\end{abstract}

Keywords: Elderly, Fall-Risk, Screening, Four Square Step Test, Unified Balance Scale, Prediction.

\section{INTRODUCTION}

Aging refers to progressive weakening of the different physical, mental psychosocial systems of the body. ${ }^{[1,2]} \mathrm{WHO}$ defines Healthy Ageing "As the process of developing and maintaining the functional ability that enables wellbeing in older age". $[3]$

In India, any person who is or above the age of 60 can be referred to as an elderly. ${ }^{[2,3]}$

There will be an exponential increase in the population of elderly in the next 50 years. Leading to an increase in the number of active adults these active adults encounter more challenging situation in daily life owning to the fact that they retire late in the life. With the increasing challenges, they are at more risk for falls.

In general falls are a major incidence in an elderly life and hence its sequelae have a major effect on their daily living.

A fall can be defined as "an unexpected event, in which the participant comes to rest on the ground, floor, or lower level" and "excludes coming to rest against furniture, wall, or other structure". ${ }^{4}$

In India, the prevalence of falls was $14 \%$ to $53 \%$ among individuals aged 60 years and Older. $^{[3]}$ Falls cause a large number of injuries which leads to an increase in mortality and morbidity. Hence, high-risk individuals should be correctly identified using screening measures for 
focused intervention. Also, it is cost efficient to forestall falls rather than treating them. ${ }^{[4,7,8,9]}$

The balance system is multisensory, incorporating inputs from the vestibular, visual, and somatosensory/proprioception systems. Hence balance is proved to be an important predictor of falls in the elderly group of people.

One previous study concluded that falls are bound to happen 7.9 times more during a turn than walking straight. Also, it was found that $35 \%$ to $45 \%$ of all steps taken are non-straight steps during walking in a typical day. Therefore, side walking and turning are important factors to predict falls. ${ }^{[11,12]}$

Four-Square Step Test (FSST) which evaluates ability to rapidly step in all four directions (forward, backward, sideways) while crossing of over a low obstacle. Furthermore, multidirectional stepping causes structural and functional changes at the central nervous system. In order prevent falls a safer, smoother, and more efficient multidirectional stepping, is needed which is owing to a flexible and adaptable motor system. They also found that stepping speed decreases with age and is lower for fallers than non-faller. ${ }^{[13,2]}$

Numerous physical performance tests have also been used for screening risk of falls among older adults like Timed Up and Go (TUG), 5 times sit-to-stand, the Berg Balance Scale and Fullerton Advanced Balance Scale, some of which are simple and focus on one aspect of balance while others are composite and cover multiple aspects of balance. The screening protocol should hence encompass all these factors which when impaired result in a fall. ${ }^{[14]}$

Unified balance scale (UBS) is a combination of three existing scales hence it can be assumed that Unified balance scale has the good properties of all the scales and would have a better chance at predicting falls in elderly.

Purpose and Need of Study: Clinicians and researchers are presented with a wide, and sometimes confusing, assortment of options, making the choice of selecting an appropriate screening tool which can most accurately identify elderly with a risk of falls in the minimum amount of time very difficult. The decision lay in front of him/her regarding using a simple and speedy test or to perform a detailed assessment for a better result. ${ }^{[15]}$

There are different studies stating that the short tests like FSST are as good as elaborate ones to identify fallers, but no direct comparison is done between FSST and the Unified Balance Scale until now.

Hence the purpose of this study was to compare a Unified balance scale(which is a detailed scale) with Four-square step test(which is a simpler test) to see which could give a better prediction of people who had a greater risk of falls from people who had lesser risk of falls.

Aims and objectives of the study: To compare and correlate the sensitivity and specificity of Unified balance scale and Four-square test.

\section{METHODOLOGY}

This is a cross-sectional analytical study performed on 80 healthy elderly above 60 years of age.

Sample size was calculated with consultation of statistician. Convenient sampling was done.

Power was set at $80 \%$ and confidence interval at $95 \%$.

\section{Inclusion criteria:}

(i) Healthy elderly, with or without assistive device.

(ii) Must be able to stand at least 5 minutes without assistance.

(iii)Able to walk minimum $12 \mathrm{~m}$ at a time.

(iv)MMSE score above 24.

\section{Exclusion criteria: Subjects with,}

(i) Diagnosed neuromuscular conditions

(ii) History of any orthopaedic injury or surgery within 3 months. 
(iii)Other Diseases that might have an influence on balance or gait.

(iv) Any problems that might impair the ability to communicate information about fall status and frequency.

\section{PROCEDURE:}

Approval was obtained from the ethics committee prior to commencement of the study. The study was explained to all participants and informed consent was taken from all subjects. The demographic data was collected as per the Case Record sheet. Participants were administered UBS and FSST and the readings were noted. Scores of the two were statistically correlated. Fall history was noted prior to the administration of the scales.

Four Square Step Test (FSST) - It is a test in which 4 canes are laid in a Criss cross pattern. The subject then completes the sequence as fast as possible. This includes that the subject starts in one square and steps in one direction into each of the four squares and then reverses direction back to the start. This makes the subject step forward, backward sideways to right and left. $^{[14]}$

Unified balance scale (UBS) - The Unified Balance scale is a scale made from items complied from the Berg Balance, FAB and POMA Tinetti. It has 5 subcomponents namely - sitting, transfers, static standing, dynamic standing, and walking. Each item has different scoring ranging from $0-1$ or 0 5 where 0 indicates lowest level of function and 5 indicates highest level of function. It is a newly developed scale which can be used for elderly. ${ }^{[15]}$

\section{STATISTICAL ANALYSIS}

Data was entered using MS Excel 2010 and analysed using SPSS16 software (Inc., Chicago, IL) for windows.

All the statistical tests were performed at the level of significance of 0.05 and confidence interval of $95 \%$.

The descriptive statistics were calculated for age, gender, number of falls in the past year.

The collected data was analysed for correlation and it was found out using Spearman Correlation test.

Data was used to plot a contingency table for sensitivity and specificity. Sensitivity of the scales was established from the table by the formula Sensitivity = True positives/True positives + False negatives.

Specificity of the scales was established from the table by the formula Specificity $=$ True negative $/$ False positive + True negative.

\section{RESULTS}

Eighty healthy elderly participated in the study. Among the participants 41 were males and 39 were females.

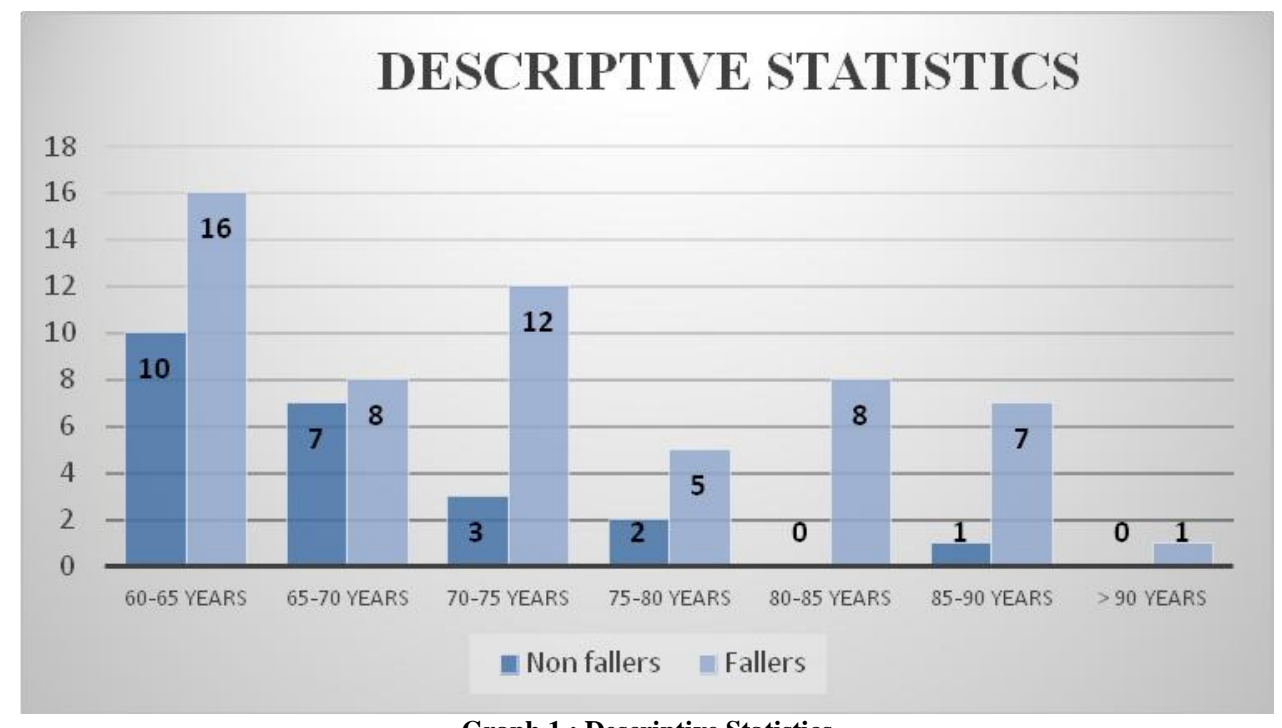

Graph 1 : Descriptive Statistics 
Arwa Sinnarwala et.al. Predicting falls in healthy elderly: a comparison between unified balance scale versus four-square step test.

Table 1: Comparison of score of fallers and non-faller between the two scales.

\begin{tabular}{|c|c|c|}
\hline Scale & $\begin{array}{l}\text { Fallers Score } \\
(\mathbf{N}=\mathbf{5 7})\end{array}$ & $\begin{array}{l}\text { Non-Fallers Score } \\
(\mathbf{N}=\mathbf{2 3})\end{array}$ \\
\hline Unified Balance Scale & $45.05 \pm 7.6$ & $54.56 \pm 5.2$ \\
\hline Four Square Step Test & $13.97 \pm 2.1$ & $10.34 \pm 2.2$ \\
\hline
\end{tabular}

On analysing the correlation, Falls and Unified Balance Scale had a significant negative correlation value at -0.683 . Which means that as the scores of UBS reduced the risk of falls increased. Whereas falls and FSST had a significant positive correlation value at 0.788 . Which means that as the time in seconds increased the risk of falls also increased.

\section{GRAPH 2: Roc Curve for Unified Balance Scale and FSST} Area under the curve : 0.92 for UBS

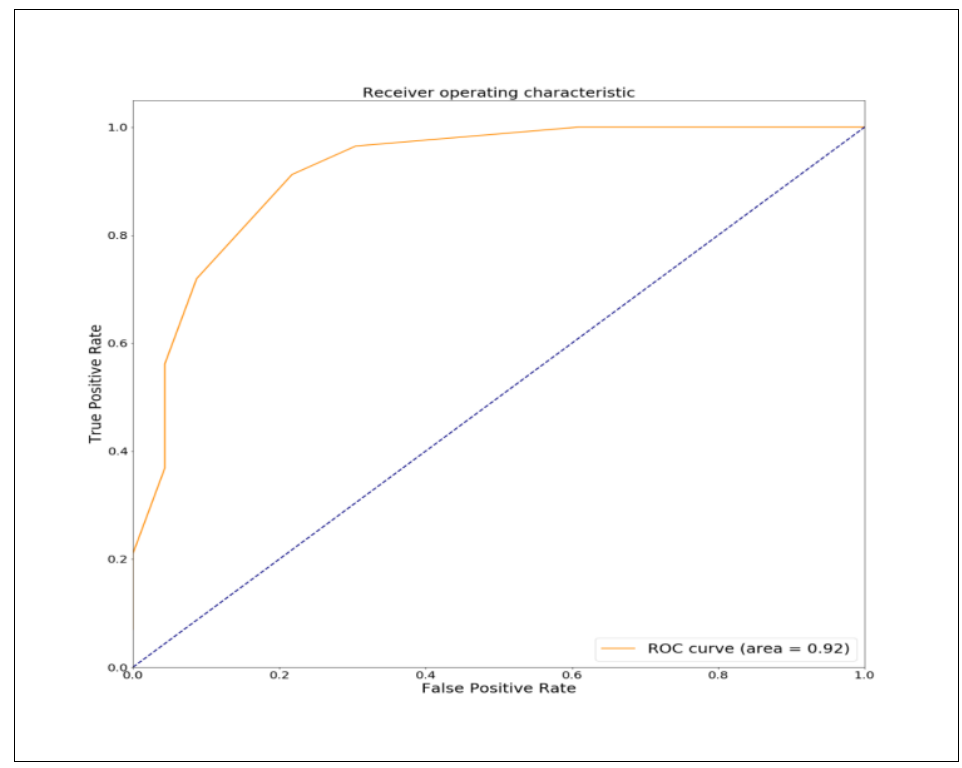

\section{Area under the curve : 0.85 for FSST}

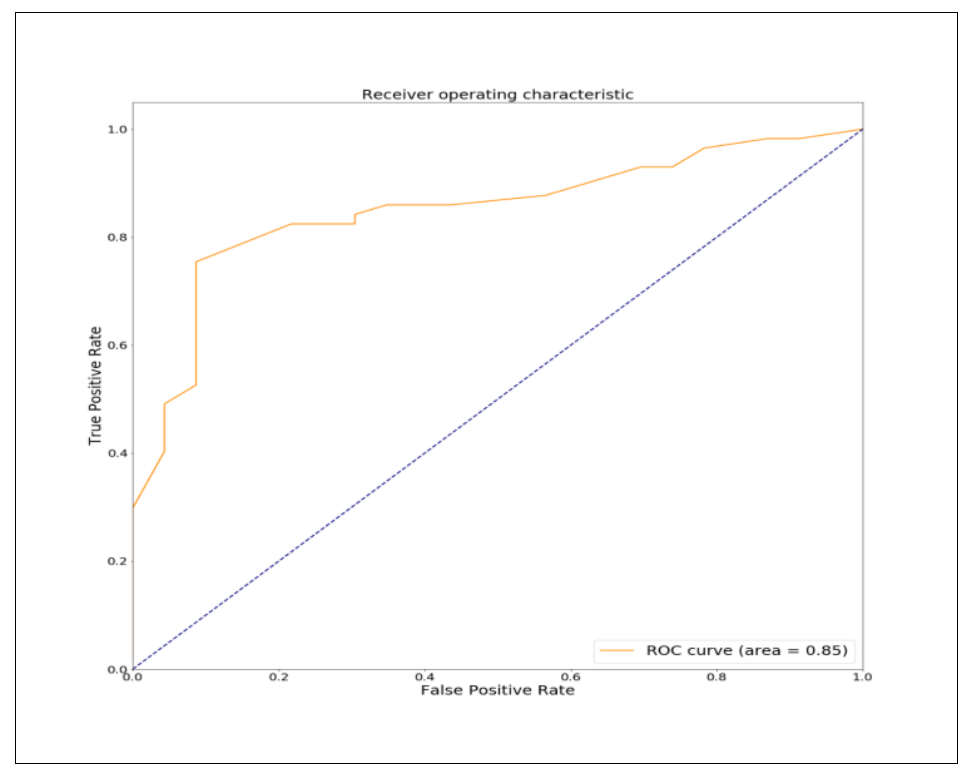

The AUC values indicated the following:

High accuracy when AUC $>0.9$,

Moderate accuracy when AUC $=0.7-0.9$,

Low accuracy when AUC $=0.5-0.69$, 
Value due to chance when AUC $<0.5 .^{[18,30]}$

Hence, we can see that UBS has high accuracy whereas FSST has moderate accuracy proving that UBS is better than FSST.

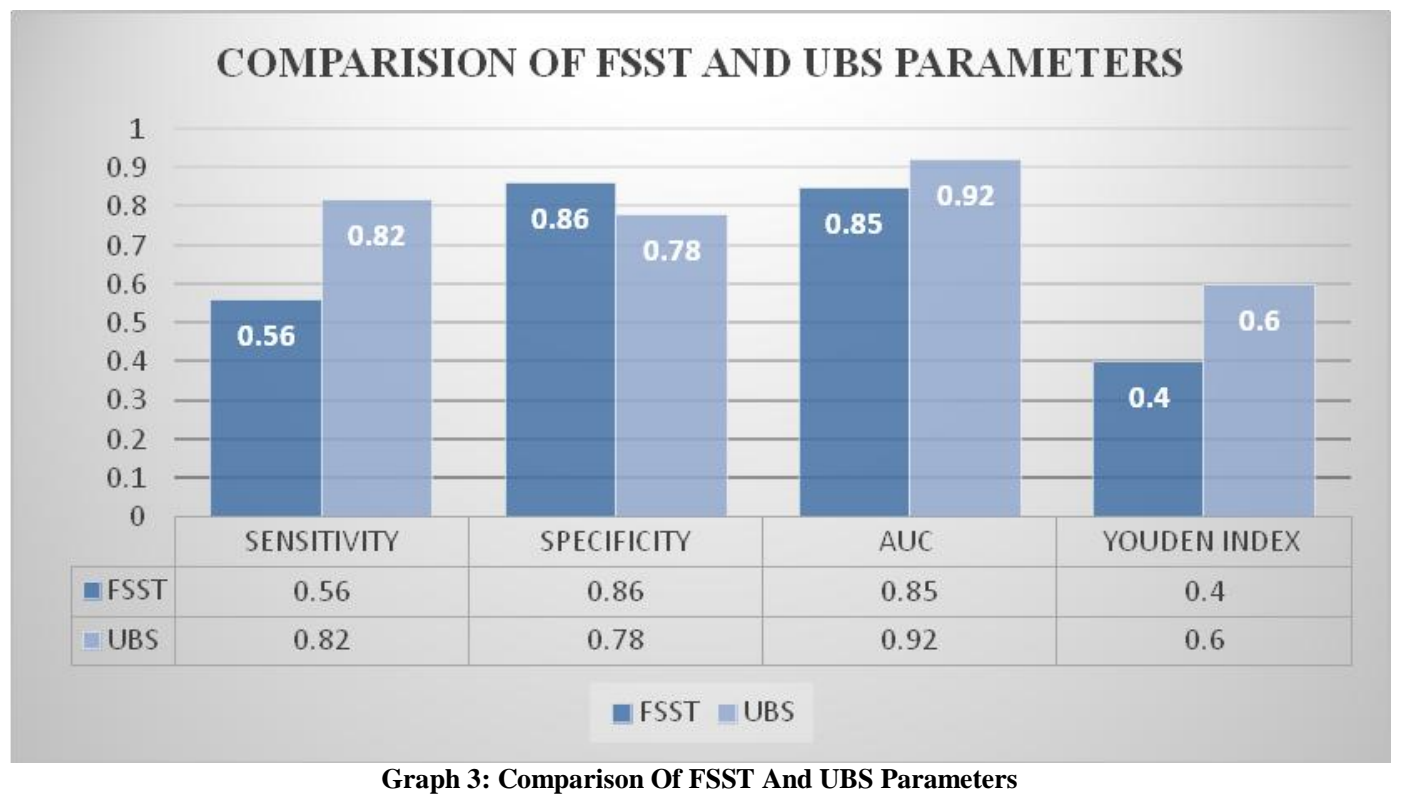

\section{DISCUSSION}

The finding of this study revealed that Unified Balance Scale had better Sensitivity and specificity and was hence better at predicting falls in healthy elderly.

A good fall risk screening instrument in healthy elderly should be short, understandable, easy to administer and at the same time should be challenging enough to distinguish between various categories of individuals with different level of strength and balance. ${ }^{[16]}$

Previous literature has shown than a low sensitivity can falsely have a person believe about the wellbeing of his health and the he is at no risk of fall. This might make him overconfident and avoid necessary help or intervention which may lead to fall. Falls also cause further fear and anxiety leading to reduced mobility. Whereas precaution and fall intervention strategies have much better prospect of avoiding such consequences hence a midway between sensitivity and specificity should be obtained. ${ }^{[5]}$

Results of this study differ from the previous studies which state that FSST score of below 14 seconds could predict community-living older adults who are at a risk of falling.

This may be since FSST is a single domain performance measure and balance includes sum of deficits across multiple domains more accurately. Earlier literature stated that even though as it is a single domain measure critical information can be lost if this domain of balance is not affect making it inaccurate to predict falls. ${ }^{[17]}$

In this study we found a lower specificity might just classify a non-faller as a faller in some cases. Having such false positive is not as severe as classifying someone as false negative. Cause having a false positive is just making the person more alert and aware of the surrounding and making themselves more equipped and prepared in incidences of falls. ${ }^{[18]}$

The results of the current study proved that a cut off score of 50 in UBS would correctly identify $82.6 \%$ of fallers (sensitivity) and $78.6 \%$ of non-fallers (specificity). Hence it can be considered for further use. ${ }^{[19]}$ The results of this study show that a simple stepping in different direction is only one aspect of balance assessment. This is in accordance with Ankur Desai and colleagues who state that other aspects of 
gait should also be assessed like stability adaptability over different surface which can also be a cause of fall. ${ }^{[20]}$

Also, Four-Square step test may have been a too easy for this healthy elder population with the high cut off score which is normally used for a low functioning elders. ${ }^{[21]}$ Hence the individuals in this study were easily able to complete it below the cut of score even though they had a fall and were identified as false negative.

To assess performance and find out balance issues a scale needs more challenging balance task which like static, dynamic, proactive, and reactive balance control. ${ }^{[22]}$ Unified balance scale includes all these components from established scales. This helps the clinician to have an overall idea of balance and help in better prediction of falls. ${ }^{[15]}$

Unified balance scale helped identifying individuals having problems with inability to adjust his/her balance during an ongoing task when suddenly placed under an unknown situation. Also, previous study showed that a person needs rapid activation of synergy and correction of balance strategy to prevent falls. ${ }^{[23,24]}$

The findings of the present study also demonstrated that UBS gave an idea about their lower limb strength and ability to transfer safely which are proved to be an identifier of falls. Also, lower limb strength helps prevent falls during postural change activities of daily living. This finding is supported by a previous study which stated that maximal voluntary isometric knee extension strength reflects the forces a muscle can produce. Kimberley S.van Schooten et al also confirmed that most falls occurred during stand to sit as involved change in potential energy. ${ }^{[25]}$

Kazunori Akizuki and colleagues reported that tasks such as perturbation or leaning movement was better in discriminating fallers from non-fallers which is in agreement with the finding of the present study. ${ }^{[24]}$

Various studies have stated that fallers having a decrease in proprioception, visual acuity, quadriceps strength and cutaneous sensation. This proves that older adults use more of vision that somatosensory system which supports our findings of UBS component of eyes closed. ${ }^{[26,27]}$

Evidence also pointed out that with age the quality and quantity of hair cells in the vestibular system decreases resulting in decreased capacity to detect rotational head movements and maintain pace along with head turns. In this study also a lower score was given to patients with reduced head turns or inability to maintain previous pace. Thus, impairments in vestibular function can affect the ability to detect changes in head acceleration and may have contributed to slowing of head-turn gait speed making them at greater risk of falls. ${ }^{[28]}$

Anacker and Fabio indicated that One-legged balance is supposedly needed to perform one's ADL's safely and it is thought to decrease with advancing age and is able to differentiate older adults who suffered multiple falls. Present study finding strengthens this aspect of including one legged balance test component to help predict fallers. ${ }^{[29]}$

Finally, the study result concluded that fallers had less stance time than nonfallers. Additionally, the stance time of faller older adults was further reduced while they were standing on a foam as compared to a normal ground this is known as sensory reweighting Hence stance time on foam surface could be a distinguishing factor between fallers and non-fallers. ${ }^{[27,30]}$

Hence this study concludes that Unified Balance Scale is better than FourSquare Step test to predict falls in healthy elderly population

\section{CONCLUSION}

Both the scales show significant correlation with Number of falls and each other

Four square step test has a sensitivity of $56 \%$ and specificity of $86 \%$ at a cut off score of 14 seconds 
Unified balance scale has a sensitivity of $82.6 \%$ and specificity of $78.6 \%$

Hence Unified balance scale is better than Four Square Step test to predict falls in healthy elderly population.

\section{Future scope:}

Scale can be used to in a 6-month, 12-month prospective design to see follow up of falls.

Scale can be used in a study consisting individuals of other conditions like Stroke and Parkinson disease to see if it can predict falls in these conditions.

\section{Acknowledgement: None}

\section{Conflict of Interest: None}

\section{Source of Funding: None}

\section{Ethical Approval: Approved}

\section{REFERENCES}

1. World Health Organization. (2015). World report on ageing and health. Geneva, Switzerland: World Health Organization.https://www.who.int/newsroom/fact-sheets/detail/ageing-andhealth

2. Chavan U, Sheikh A. Effect of Stability Trainer Exercise Program on Balance in Geriatric Population. J Community Med Health Educ 2017;7:2.

3. https://www.who.int/ageing/healthyageing/en/

4. Dsouza SA, Rajashekar B, Dsouza H, Kumar K. Falls in Indian older adults: a barrier to active ageing. Asian J Gerontol Geriatr 2014;9:1-8.

5. Martins AC, Moreira J, Silva C, Silva J, Tonelo C, Baltazar D, Rocha C, Pereira T, Sousa I. Multifactorial screening tool for determining fall risk in community-dwelling adults aged 50 years or over (FallSensing): Protocol for a prospective study. JMIR research protocols. 2018;7(8):e10304.

6. 8.World Health Organization (2012) Fact sheet 344: Falls. http:// www.who.int/mediacentre/factsheets/fs344/ en/. Accessed 6 June 2015)
7. Kamide N, Shiba Y, Sakamoto M, Sato H, Kawamura A. Fall-related efficacy is a useful and independent index to detect fall risk in Japanese community-dwelling older people: a 1-year longitudinal study. BMC Geriatr 2019;19:293. https://doi.org/10.1186/s12877-019-1318-5

8. Pfortmueller CA, Lindner G, Exadaktylos AK. Reducing fall risk in the elderly: risk factors and fall prevention, a systematic review. Minerva Med. 2014 Aug 1;105(4):275-81.69

9. Criter RE, Honaker JA. Identifying balance measures most likely to identify recent falls. Journal of geriatric physical therapy. 2016 Jan 1;39(1):30-7.

10. Carter V, Jain T, James J, Cornwall M, Aldrich A, de Heer HD. The 3-m backwards walk and retrospective falls: diagnostic accuracy of a novel clinical measure. Journal of Geriatric Physical Therapy 2019;42:249-55.

11. Gamerman Y, Hoshen M, Herman AC, Alter Z, Hadad L, Melzer I. Validity and Interobserver Reliability of the TURN 180 Test to Identify Older Adults Who Reported Falls. The Israel Medical Association Journal: IMAJ 2019;21:269-74.

12. Glaister BC, Bernatz GC, Klute GK, Orendurff MS. Video task analysis of turning during activities of daily living. Gait Posture 2007;25:289-94.

13. Giannouli E, Morat T, Zijlstra W. A novel square-stepping exercise program for older adults (StepIt): rationale and implications for falls prevention. Frontiers in Medicine 2020;6:318.70

14. Langford $Z$. The four square step test. Journal of physiotherapy. 2015 Jul 1;61(3):162.

15. La Porta F, Franceschini M, Caselli S, Cavallini P, Susassi S, Tennant A. Unified Balance Scale: an activity-based, bed to community, and aetiology-independent measure of balance calibrated with Rasch analysis. Journal of Rehabilitation Medicine 2011;43:435-44.

16. Reider NC, Naylor PJ, Gaul C. Sensitivity and specificity of the minimal chair height standing ability test: a simple and affordable fall-risk screening instrument. Journal of geriatric physical therapy. 2015 Apr 1;38(2):90-5.

17. Persad C, Cook S, Giordani B. Assessing falls in the elderly: should we use simple 
screening tests or a comprehensive fall risk evaluation? European Journal of Physical and Rehabilitation Medicine 2010;46:24959.

18. Kim T, Xiong S. Comparison of seven fall risk assessment tools in communitydwelling Korean older women. Ergonomics 2017;60:421-9.

19. Perell KL, Nelson A, Goldman RL, Luther SL, Prieto-Lewis N, Rubenstein LZ. Fall risk assessment measures: an analytic review. J Gerontol A Biol Sci Med Sci 2001;56:M761-6.

20. Desai A, Goodman V, Kapadia N, Shay BL, Szturm T. Relationship between dynamic balance measures and functional performance in community-dwelling elderly people. Physical Therapy 2010;90:748-60.

21. Balasubramanian CK, Boyette A, Wludyka P. How well do functional assessments of mobility and balance discriminate fallers and recurrent fallers from non-fallers among ambulatory older adults in the community? Physiotherapy Canada 2015;67:184-93.

22. Lee J, Geller AI, Strasser DC. Analytical review: focus on fall screening assessments. PM\\&R 2013;5:609-21.

23. Brauer SG, Burns YR, Galley P. A prospective study of laboratory and clinical measures of postural stability to predict community-dwelling fallers. The Journals of Gerontology Series A: Biological Sciences and Medical Sciences 2000;55:M469M47657.

24. Akizuki K, Echizenya Y, Kaneno T, Ohashi $Y$. Usefulness of an unstable board balance test to accurately identify communitydwelling elderly individuals with a history of falls. Journal of Rehabilitation Medicine 2019;51:71-6.

25. Van Schooten KS, Yang Y, Feldman F, Leung M, McKay H, Sims-Gould J, et al. The Association between fall frequency, injury risk, and characteristics of falls in older residents of long-term care: Do recurrent fallers fall more safely? The Journals of Gerontology: Series A 2018; 73:786-91.

26. Pizzigalli L, Cremasco MM, Mulasso A, Rainoldi A. The contribution of postural balance analysis in older adult fallers: a narrative review. Journal of Bodywork and Movement Therapies 2016;20:409-17.

27. Aartolahti E, Häkkinen A, Lönnroos E, Kautiainen H, Sulkava R, Hartikainen S. Relationship between functional vision and balance and mobility performance in community-dwelling older adults. Aging Clin Exp Res 2013;25:545-52. https://doi.org/10.1007/s40520-013-0120-z.

28. Singh H, Sanders O, Waller SM, Bair W-N, Beamer B, Creath RA, et al. Relationship between head-turn gait speed and lateral balance function in community-dwelling older adults. Archives of Physical Medicine and Rehabilitation 2017;98:1955-61.

29. Moreira MN, Bilton TL, Dias RC, Ferriolli E, Perracini MR. What are the main physical functioning factors associated with falls among older people with different perceived fall risk? Physiotherapy Research International 2017;22:e1664.75

30. Mathurapongsakul P, Siriphorn A. Four Square Step Test With Foam Is More Accurate Than Those Without Foam for Discriminating Between Older Adults With and Without Fall History. J Aging Phys Act 2018;26:624-8. https://doi.org/10.1123/japa.2017-0363.

How to cite this article: Sinnarwala A, Joshi S. Predicting falls in healthy elderly: a comparison between unified balance scale versus foursquare step test. Int J Health Sci Res. 2021; 11(7):1-8. DOI: https://doi.org/10.52403/ijhsr. 20210701 